\title{
Cloning Laboratory Shared Resource
}

National Cancer Institute

\section{Source}

National Cancer Institute. Cloning Laboratory Shared Resource. NCI Thesaurus. Code C39363.

The Cloning Laboratory Shared Resource provides restriction enzymes, DNA and RNA modification enzymes, host cell lines, kits, cloning and expression vectors, competent cells, molecular weight markers, and other chemicals and reagents for Cancer Center research projects in the fields of molecular biology and biochemistry. 